\title{
Alveolar lining fluid regulates mononuclear phagocyte 5-lipoxygenase metabolism
}

\author{
S.M. Phare, M. Peters-Golden, M.J. Coffey
}

Alveolar lining fluid regulates mononuclear phagocyte 5-lipoxygenase metabolism. S.M. Phare, M. Peters-Golden, M.J. Coffey. (OERS Journals Ltd 1998.

ABSTRACT: The enzyme 5-lipoxygenase (5-LO) catalyses the synthesis of leukotrienes (LT), which are important in phagocytosis and killing of microorganisms. The alveolar macrophage (AM), the primary resident defender of the alveolar space, has a greater capacity for LT synthesis than its precursor, the peripheral blood monocyte (PBM). This study investigated whether the alveolar lining fluid (ALF) upregulates LT synthetic capacity in mononuclear phagocytes.

Rat AM, peritoneal macrophages (PM) and ALF were obtained by lavage from pathogen-free animals. Human PBM were isolated from normal subjects. 5-LO metabolism and expression were measured with and without ALF.

Rat ALF increased 5-LO metabolism (136.4 $\pm 15.1 \%$ of control) in cultured PBM. This was associated with increased 5-LO activating protein (FLAP) $(357 \pm 29.5 \%)$, and 5-LO expression $(188 \pm 31.3 \%)$. Culture of $A M$ for 3 days resulted in a greater decrement in $\mathrm{LTB}_{4}$ synthesis $\left(\mathrm{LTB}_{4} \mathbf{1 5 . 4 \pm 6 . 9 \%}\right.$ of day 1$)$ than in PM $(54.7 \pm 8.3 \%$ of day 1$)$, suggesting a greater dependence of AM 5-LO metabolism on ALF. 5-LO and FLAP expression decreased to a greater degree in AM than PM in culture. Furthermore, AM cultured with ALF maintained their LT synthetic capacity, FLAP and 5-LO expression compared with control cells cultured in medium alone.

In conclusion, alveolar lining fluid increased 5-lipoxygenase metabolism in peripheral blood monocytes and maintained it in cultured alveolar macrophages, by a mechanism of increased 5-lipoxygenase and 5-lipoxygenase activating protein expression. This may boost host defence capabilities.

Eur Respir J 1998; 12: 1141-1146.

Arachidonic acid (AA), which is hydrolysed from membrane phospholipids by phospholipase $\mathrm{A}_{2}$, is converted to leukotrienes (LT) by the enzyme 5-lipoxygenase (5-LO), with the aid of 5-LO activating protein (FLAP) [1]. The main 5-LO products of AA are $\mathrm{LTB}_{4}, \mathrm{LTC}_{4}$, and 5-hydroxyeicosatetranoic acid (5-HETE) and these have been implicated in a number of disease states, including pulmonary diseases such as asthma [2] and interstitial lung disease $[3,4]$. The cell with the greatest capacity for LT synthesis in the lung is the alveolar macrophage (AM). This cell is the main resident pulmonary immune effector cell and serves to defend the alveolar space against foreign antigens and microbes [5, 6].

AM are derived from peripheral blood monocytes (PBM), which migrate into the lung and undergo a process of differentiation into mature macrophages. It has been previously demonstrated that the AM has a greater capacity for LT synthesis than its precursor, the PBM [7-9], or other nonpulmonary macrophages, e.g. the peritoneal macrophage (PM) [10]. The increased 5-LO metabolic capacity of AM as compared to PBM can be attributed primarily to an increased level of FLAP expression, and to a lesser extent to increased 5-LO expression [9]. The finding that interstitial pulmonary macrophages share with PBM a lim-ited capacity for LT synthesis indicates that the high LT synthetic capacity of AM only develops upon
Divisions of Pulmonary and Critical Care Medicine, University of Michigan, Medical Center, Ann Arbor, Michigan, USA

Correspondence: M.J. Coffey

University of Michigan Medical Center

6301 MSRB III

1150 W. Medical Center Drive

Ann Arbor

MI 48109-0642

USA

Fax: 17347644556

Keywords: Eicosanoid

leukotriene

lung

macrophage

monocyte

Received: January 191997

Accepted after revision July 21998

Supported by a grant from the American Lung Association of Michigan. M.J. Coffey was the recipient of National Institutes of Health Clinical Investigator Development Award. entry into the alveolar space [10]. It has also been reported previously that the AM LT synthetic capacity declines over time in culture, suggesting that the maintenance of 5-LO metabolism is dependent on factors present in the alveolar environment [11].

These observations led to the hypothesis that factors in the alveolar space may be important in the upregulation of 5-LO metabolism in PBM as they move into the lung and differentiate into AM. Furthermore, the alveolar environment may be essential for the maintenance of AM LT synthetic capacity. Therefore, this study examined the effect of alveolar lining fluid (ALF) on mononuclear phagocyte 5-LO metabolism. Initially, the capacity of ALF to upregulate 5-LO metabolism in PBM was examined. Subsequently, the role of ALF in maintaining the high level of 5-LO metabolism in AM was investigated.

\section{Methods}

Isolation of human peripheral blood monocytes and rat alveolar and peritoneal macrophages

Human PBM were isolated from venous blood drawn from normal volunteers. Cells were purified by a magnetic cell sorter (Miltenyi Biotec, Auburn, CA, USA), as described previously [12]. Cells prepared by this method have consistently been shown to be quiescent, as judged by a 
lack of eicosanoids generated in the absence of an exogenous stimulus. This method yielded PBM that were $>97 \%$ pure by differential staining and $>95 \%$ viable, as indicated by trypan blue exclusion. The rat AM was studied because it is a good model of human AM 5-LO metabolism, and has been well characterized in the authors' laboratory [10, 13]. Rat PM were studied as a model of nonpulmonary macrophages, since rat PBM are difficult to obtain in sufficient numbers to study. AM and PM were obtained from $150 \mathrm{~g}$ specific pathogen-free female Wistar rats, as described previously [13]. For the isolation of cells, lavage buffer consisting of $0.15 \mathrm{M} \mathrm{NaCl}, 2.7 \mathrm{mM}$ ethylenediaminetetraacetic acid (EDTA), $20 \mathrm{mM}$ HEPES, $5.5 \mathrm{mM}$ dextrose, $100 \mathrm{U} \cdot \mathrm{mL}^{-1}$ penicillin, $100 \mu \mathrm{g} \cdot \mathrm{mL}^{-1}$ streptomycin and $0.25 \mathrm{mg} \cdot \mathrm{mL}^{-1}$ amphotericin B was used. In order to obtain resident AM, rats were anaesthetized with sodium pentobarbital i.p. and the lungs were excised and lavaged in 5$\mathrm{mL}$ aliquots with a total of $50 \mathrm{~mL}$ cold lavage fluid. Lavaged fluid was centrifuged at $500 \mathrm{~g}$ for $10 \mathrm{~min}$ at $4{ }^{\circ} \mathrm{C}$ and the cell pellet resuspended in $\mathrm{Ca}^{2+}-$ free and $\mathrm{Mg}^{2+}$-free Dulbecco's phosphate-buffered saline (PBS). Microscopic examination of cytocentrifuge preparations stained with a modified Wright-Giemsa stain (Diff-Quick; American Scientific Products, McGraw Park, IL, USA), revealed that the cells were $>90 \%$ AM, and $>98 \%$ AM following adherence. Viability was $>95 \%$, as assessed by trypan blue exclusion.

Resident PM were obtained from separate animals, which were anaesthetized with ether. After a superficial midline incision, the skin overlying the abdomen and thorax was reflected laterally and the animals were exsanguinated by cutting a subclavian vessel. Peritoneal lavage was performed by the sequential injection into the peritoneum and aspiration of three $10-\mathrm{mL}$ aliquots of iced lavage fluid containing $10 \mathrm{U} \cdot \mathrm{mL}^{-1}$ of heparin. Recovered fluid was pooled and centrifuged at $500 \mathrm{~g}$ for $10 \mathrm{~min}$ at $4^{\circ} \mathrm{C}$. Erythrocytes in the cell pellet were hypotonically lysed, tonically restored with $2 \times \mathrm{PBS}$ and resuspended for counting in a haemocytometer. Microscopic examination of cytocentrifuge preparations stained with Wright-Giemsa indicated that $\sim 81 \%$ of lavaged cells were macrophages, increasing to $>98 \%$ pure with adherence, and $>95 \%$ viable, as ass-essed by trypan blue exclusion [14].

\section{Alveolar lining fluid}

To obtain ALF diluted in a standard manner, rats were lavaged as described above with $10 \mathrm{~mL}$ of sterile lipopolysaccharide (LPS)-free Dulbecco's modified Eagles medium (DMEM) in place of the lavage buffer used to isolate AM. This fluid is a best approximate for ALF, but does also contain fluid from the large and small airways. Lavaged cells were pelleted by centrifugation and the supernatant was subsequently filtered $(0.2 \mu \mathrm{m})$ to ensure that it was sterile for culturing cells in vitro.

\section{Cell-culture conditions.}

Isolated PBM, AM and PM were resuspended in LPS-free DMEM at $0.5 \times 10^{6} \cdot \mathrm{mL}^{-1}$ and plated as follows: $0.2 \mathrm{~mL} \cdot$ well $^{-1}$ in 96 -well plates for enzyme immunoassay (EIA), $1 \mathrm{~mL} \cdot$ well-1 $^{-1}$ in 24 -well plates for $\left[{ }^{3} \mathrm{H}\right] \mathrm{AA}$ release studies, and $5 \mathrm{~mL} \cdot 35 \mathrm{~mm}^{-1}$ culture plate for immunoblot analysis. Cells were cultured in DMEM containing antibiotics penicillin G, streptomycin and amphotericin B. Cells were incubated in the presence of $10 \%$ foetal calf serum (FCS), with the exception of experiments examining the role of ALF on 5-LO metabolism. Serum was not utilized in these experiments because several factors in serum, such as vitamin $D_{3}$ and transforming growth factor- $\beta$ (TGF- $\beta$ ), are known to upregulate 5-LO metabolism and this could complicate the interpretation of the results. Cells were adhered for $1 \mathrm{~h}$ at $37^{\circ} \mathrm{C}$ in a humidified atmosphere of $5 \% \mathrm{CO}_{2} / 95 \% \mathrm{O}_{2}$. Nonadherent cells were removed by washing twice with DMEM and adherent cells were cultured in DMEM, with or without ALF for varying periods. In these studies the viability of AM at 3 days in culture was $91 \pm 5.9 \%$ as determined by trypan blue exclusion. This is consistent with previously published data [11].

\section{Quantification of 5-lipoxygenase metabolism in intact cells}

The maximal capacity for 5-LO metabolism in intact cells was measured by EIA determination in cell-free supernatants of the predominant 5-LO product, $\mathrm{LTB}_{4}$, after stimulation of cells with ionophore A23187 $(10 \mu \mathrm{M})$ and/ or exogenous AA $(50 \mu \mathrm{M})$. Stimulation with A23187, along with exogenous substrate AA, resulted in the optimal synthesis of LT in PBM. This can be explained by the lower expression of FLAP in PBM than in AM, resulting in less efficient conversion of released endogenous AA to 5-LO products [9]. The eicosanoid profile was determined by quantifying ${ }^{3} \mathrm{H}$-radiolabelled eicosanoids (thromboxane $\mathrm{B}_{2}$, prostaglandin $\mathrm{E}_{2}\left(\mathrm{PGE}_{2}\right) \mathrm{LTB}_{4}, \mathrm{LTC}_{4}$ and 5-HETE) released from endogenously released $\left[{ }^{3} \mathrm{H}\right] \mathrm{AA}$ by the incubation of prelabelled cells for 30 min with A23187 (10 $\mu \mathrm{M})$, as described previously [13]. To assess AA release, cells were stimulated in the presence of $0.1 \%$ bovine serum albumin (BSA) and the radioactivity in the medium was determined [10].

Immunoblot analysis for 5-lipoxygenase and 5-lipoxygenase activating protein

The quantities of cellular 5-LO and FLAP proteins were determined by western blot analysis. Crude lysates were prepared as described previously [13] and subjected to sodium dodecyl sulphate-polyacrylamide gel electrophoresis (SDS-PAGE) by the method of LAEMML [15] on $10 \%$ acrylamide gels. Proteins were transferred to nitrocellulose membranes overnight, and probed with rabbit polyclonal antibody against either human leukocyte 5-LO (1:3,000 dilution) or amino acid residues $41-52$ of the human FLAP sequence (1:5,000 dilution) [14]. Antisera against 5-LO and FLAP were kindly provided by J. Evans, Merck Frosst (Pointe Claire-Dorval, Quebec, Canada). After washing, blots were incubated for $1 \mathrm{~h}$ with horseradish peroxidase-conjugated anti-rabbit immunoglobulin (Ig) G (Amersham, Little Chalfont, Bucks, UK) at a dilution of 1:5,000. Membranes were then washed and incubated for $1 \mathrm{~min}$ with chemiluminescence detection reagents (ECL; Amersham) and exposed to film for varying periods to ensure that densitometric quantification was performed under conditions in which band density and exposure time were linearly related. Video densitometry was performed using Image software (National Institutes of Health, Bethesda, MD, USA). 


\section{Data analysis}

Data are expressed as mean \pm SEM. Intergroup differences were analysed by analysis of variance (ANOVA), with statistical significance assessed by the Scheffé test; $p<0.05$ was considered significant.

\section{Results}

Effect of alveolar lining fluid on peripheral blood monocyte arachidonic acid metabolism

PBM are the circulating precursors of tissue macrophages. To explore the capacity of factors in the alveolar environment to upregulate 5-LO metabolic capacity, the effect of ALF on PBM 5-LO metabolism was examined. Figure 1 demonstrates that maximal $\mathrm{LTB}_{4}$ synthetic capacity following stimulation with A23187 $(10 \mu \mathrm{M})$ and exogenous AA (50 nm) for 30 min was increased in PBM following overnight culture in ALF, as compared to the control medium. Similar results were obtained following stimulation with A23187 alone (data not shown). This increase in PBM 5-LO metabolism was maintained after culture in ALF for 3 days. One possible explanation for the increase in PBM 5-LO metabolism was an increase in the release of the substrate AA from membrane phospholipids. However, ALF-treated PBM prelabelled overnight with $\left[{ }^{3} \mathrm{H}\right] \mathrm{AA}$ did not release greater levels of the substrate AA upon stimulation, and this therefore cannot explain the increased LT synthesis (table 1).

Effect of alveolar lining fluid on peripheral blood monocyte 5-lipoxygenase and 5-lipoxygenase activating protein expression

Another possible mechanism that could account for the increased LT synthetic capacity in PBM incubated with ALF is an alteration in the expression of the two proteins essential for LT synthesis, 5-LO and FLAP. Therefore, the

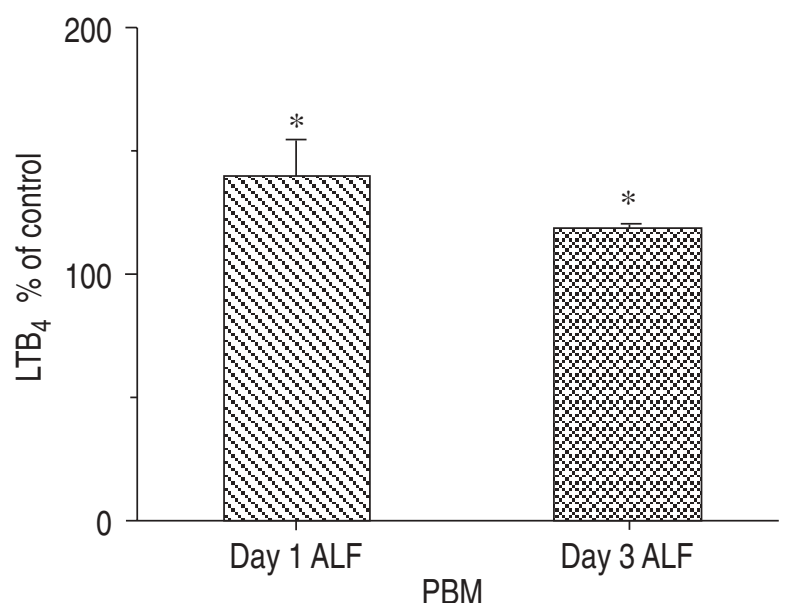

Fig. 1. - Effect of alveolar lining fluid (ALF) on leukotriene (LT) $B_{4}$ synthesis from peripheral blood monocytes (PBM) cultured for 1 and 3 days. PBM were incubated with or without ALF in the absence of serum for 1 and 3 days. The cells were then washed and incubated for $30 \mathrm{~min}$ with A23187 $(10 \mu \mathrm{M})$ and exogenous arachidonic acid $(\mathrm{AA} ; 50 \mu \mathrm{M})$. Medium was analysed for $\mathrm{LTB}_{4}$ by enzyme immunoassay and the values were expressed as a percentage of the value observed in control cells cultured in the absence of ALF (PBM day 1: $250.5 \pm 105.7 \mathrm{pg} \cdot \mathrm{mL}^{-1}, \mathrm{n}=3$; day 3: $\left.173.6 \pm 19.2 \mathrm{pg} \cdot \mathrm{mL}^{-1}, \mathrm{n}=3\right)$. *: $\mathrm{p}<0.05$ compared with control cells.
Table 1. - Effects of alveolar lining fluid (ALF) on total arachidonic acid (AA) release in peripheral blood monocytes (PBM) and alveolar macrophages (AM)

\begin{tabular}{lcc}
\hline & \multicolumn{2}{c}{ AA release (\% incorporated) } \\
\cline { 2 - 3 } & Control & ALF \\
\hline PBM & $3.12 \pm 0.87$ & $2.83 \pm 1.7$ \\
AM & $1.15 \pm 0.14$ & $1.14 \pm 0.1$ \\
\hline
\end{tabular}

Values are mean $\pm \operatorname{SEM}(n=3)$. PBM and AM were isolated and cultured with or without ALF in the absence of serum overnight. Prelabelled PBM were incubated with A23187 $(10 \mu \mathrm{M})$ and exogenous AA $(50 \mu \mathrm{M})$; and AM with A23187 $(10 \mu \mathrm{M})$ for $30 \mathrm{~min}$. Cells were stimulated in the presence of $0.1 \%$ bovine serum albumin. ${ }^{3} \mathrm{H}[\mathrm{AA}]$ was identified by co-elution with authentic standards and expressed as a percentage of incorporated radioactivity. ALF and control values were not significantly different.

expression of these proteins was determined in PBM incubated overnight with or without ALF. Figure 2 demonstrates that the expression of FLAP and, to a lesser extent, 5 -LO was increased in PBM incubated with ALF. The increased expression of these two proteins can account for the increase in LT synthetic capacity in PBM cultured in ALF.

\section{Effect of culture in vitro on alveolar and peritoneal mac- rophage 5-lipoxygenase metabolism}

Previously published studies have demonstrated that AM 5-LO metabolism decreases over time in culture [11]. To examine the relative dependence of AM 5-LO metabolism

a)
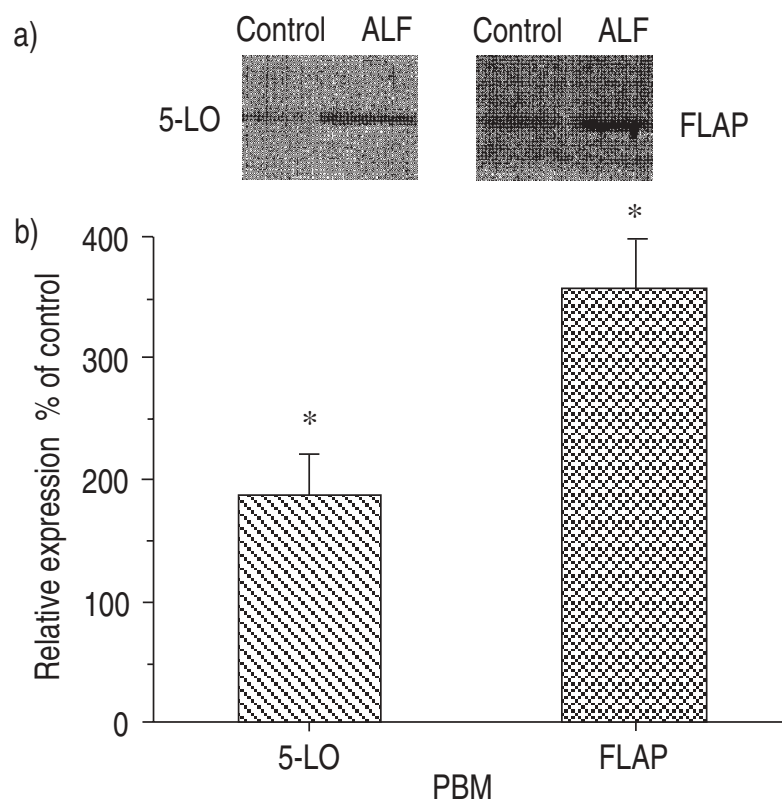

Fig. 2. - Effect of alveolar lining fluid (ALF) on 5-lipoxygenase (5LO) and 5-LO activating factor (FLAP) expression in peripheral blood monocytes (PBM). PBM were cultured with or without ALF overnight in the absence of serum. Equal amounts $(20 \mu \mathrm{g}$ protein) of crude cellular lysate from PBM were subjected to immunoblot analysis for 5-LO and FLAP. a) Representative autoradiograph of a Western blot demonstrating the amount of 5-LO (left) and FLAP (right) from control and ALF-treated PBM. b) Comparison of the relative expression of 5-LO and FLAP in PBM cultured with and without ALF, as assessed by densitometry and expressed as a percentage of values derived from control cells run in parallel. Data represent the mean \pm SEM from three experiments. *: $\mathrm{p}<0.05$ compared with control cells. 
on factors present in the alveolar space, 5-LO metabolism and 5-LO and FLAP expression were compared in AM and PM cultured over time. LT synthetic capacity decreased in both cells, but it did so in AM to a greater degree than in PM (table 2). While FLAP expression decreased over time in both cell types, it also decreased to a greater degree in AM than in PM (fig. 3). There was a trend towards a greater reduction in 5-LO expression over time in AM than PM, but this did not reach statistical significance. These findings are consistent with the conclusion that the absence of factors unique to the alveolar environment, to which the AM is exposed in vivo but which are missing in tissue culture, allow FLAP expression, and hence 5-LO metabolic capacity, to decline.

\section{Effect of alveolar lining fluid on alveolar macrophage arachidonic acid metabolism}

The next step was to examine the effect of ALF on AM 5-LO metabolism. Although AM LT synthetic capacity decreased over time in culture, incubation with ALF overnight increased, or protected from decline, 5-LO metabolic capacity compared with control AM (fig. 4). This effect was also observed after 3 days in culture. AM were prelabelled overnight with $\left[{ }^{3} \mathrm{H}\right] \mathrm{AA}$ to examine the effect of ALF on AA release as a possible cause for this increase in 5-LO metabolism. As demonstrated in table 1, AM AA re-lease was not affected by ALF, suggesting that other mech-anisms were responsible for this increase in LT synthetic capacity in ALF-treated AM.

Effect of alveolar lining fluid on alveolar macrophage 5lipoxygenase and 5-lipoxygenase activating protein expression

One possible mechanism for the increase in AM LT synthetic capacity by ALF might be through an increase in 5LO and FLAP expression, as was shown in PBM. Therefore, AM were cultured with and without ALF overnight and the expression of these proteins was assessed by Western blot analysis. Although AM 5-LO and FLAP expression in culture decreased over time, incubation with ALF in-creased FLAP in AM compared to cells incubated without ALF (fig. 5). This effect of ALF on AM FLAP expression was maintained $(269 \pm 135 \%$ of control $)$ after 3 days of culture. There was no significant effect on 5-LO

Table 2. - Effects of culture over time on alveolar macrophage (AM) and peritoneal macrophage (PM) 5-lipoxygenase metabolism

\begin{tabular}{lccc}
\hline & \multicolumn{2}{c}{$\mathrm{LTB}_{4} \mathrm{pg} \cdot \mathrm{mL}^{-1}$} & Day 3 \\
\cline { 2 - 3 } & Day 1 & Day 2 & \\
\hline AM & $2724.8 \pm 426.3$ & $475.4 \pm 280.2$ & $15.4 \pm 6.9^{*}$ \\
PM & $186.2 \pm 135$ & $114.5 \pm 85.1$ & $54.7 \pm 8.3$
\end{tabular}

Values are mean $\pm \operatorname{SEM}(n=3)$. Rat AM and PM were isolated and cultured in Dulbecco's modified Eagles medium containing $10 \%$ foetal calf serum for up to 3 days. The cells were washed and subsequently stimulated with A23187 $(10 \mu \mathrm{M})$ for $30 \mathrm{~min}$. Leukotriene (LT) $\mathrm{B}_{4}$ levels were quantitated by en-zyme immunoassay determination in the cell-free supernatants. In additions, day 3 product formation was expressed as a percentage of day 1 product formation. *: $\mathrm{p}<0.05$, comparing AM and PM on day 3 as a $\%$ of day 1 .
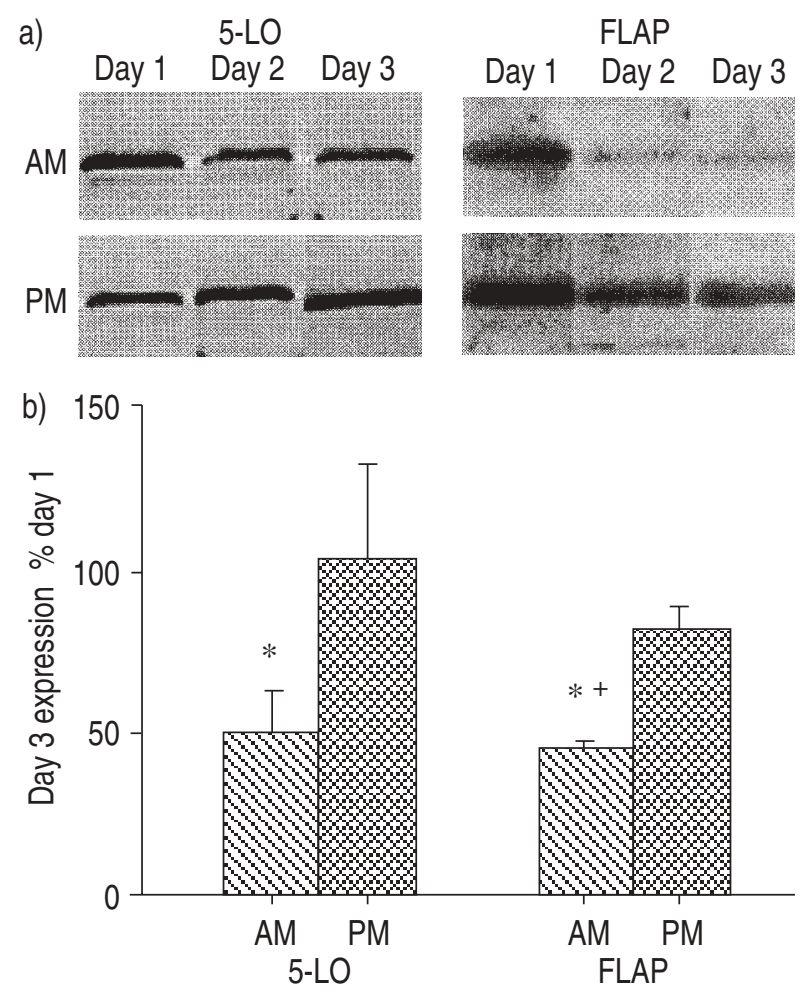

Fig. 3. - 5-lipoxygenase (5-LO) and 5-LO activating factor (FLAP) expression in alveolar macrophages (AM) and peritoneal macrophages (PM) cultured over time. Rat AM and PM were isolated and cultured over 3 days in Dulbecco's modified Eagles medium containing $10 \%$ foetal calf serum. Equal amounts of crude cellular lysate were subjected to immunoblot analysis, as described in figure 2. a) Representative autoradiograph of a Western blot demonstrating the amount of 5-LO and FLAP in AM (top) and PM (bottom) cultured over time. b) Comparison of the relative expression of 5-LO and FLAP in AM and PM on day 3 in culture compared with day $1 . *$ : $p<0.05$ compared with cells on day 1 in culture; +: p<0.05 compared with day 3 PM FLAP expression.

expression. This evidence suggests that maintenance of FLAP expression is the most likely mechanism by which ALF maintains 5-LO metabolism in cultured AM. Interestingly, there was no effect of ALF on PM 5-LO metabolism or FLAP expression (data not shown). This suggests that the effect of ALF on 5-LO metabolism is specific for undifferentiated monocytes or terminally differentiated macrophages which were exposed to ALF in vivo.

\section{Discussion}

In this study, evidence is provided that: 1) factors in ALF are instrumental in increasing 5-LO metabolism in PBM through the mechanism of increased 5-LO and FLAP expression; 2) LT synthetic capacity and FLAP expression decline to a greater degree in AM cultured ex vivo than in nonpulmonary macrophages; and 3) maintenance of the high level of AM 5-LO metabolism is dependent on factors in ALF which augment FLAP expression.

The AM has been shown in a number of studies to display a greater capacity for LT synthesis than its precursor, the PBM [8-10]. Nonpulmonary mononuclear phagocytes synthesize predominantly prostaglandins and have greater expression of the enzyme cyclo-oxygenase than do AM [16]. In contrast, they synthesize smaller amounts of LT. The mechanism for this difference in 5-LO metabolism 


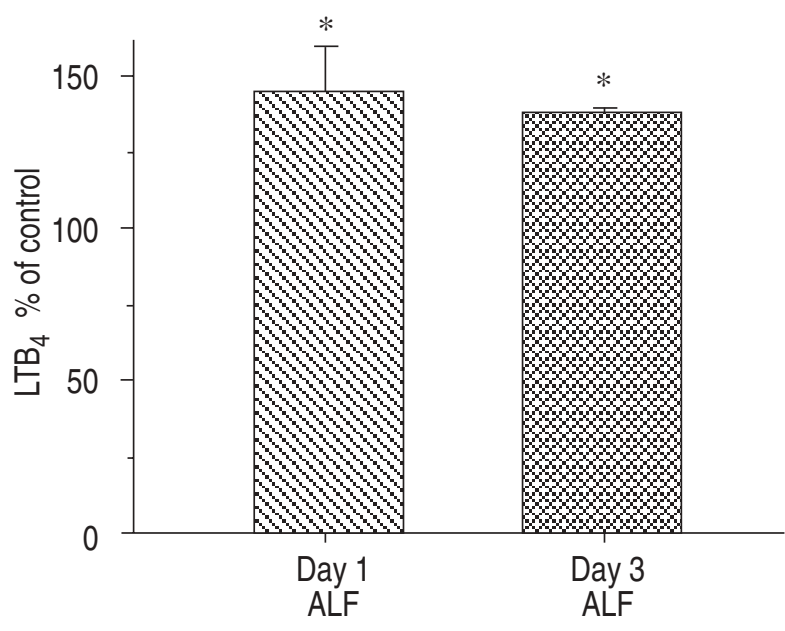

Fig. 4. - Effect of alveolar lining fluid (ALF) on leukotriene (LT) $B_{4}$ synthesis from alveolar macrophages (AM) cultured for 1-3 days. AM were incubated with or without ALF in the absence of serum for 1-3 days. The cells were then washed and incubated for 30 min with A23187 $(10 \mu \mathrm{M})$. The medium was analysed for $\mathrm{LTB}_{4}$ by enzyme immunoassay and the values were expressed as a percentage of the value observed in control cells cultured in the absence of ALF (AM day 1: 2,486.9 \pm 190 $\mathrm{pg} \cdot \mathrm{mL}^{-1}$; day 3: $\left.652.1 \pm 312.0 \mathrm{pg} \cdot \mathrm{mL}^{-1}, \mathrm{n}=3\right)$. *: $\mathrm{p}<0.05$ compared with control cells.

was demonstrated to be a greater expression of FLAP and, to a lesser extent, 5-LO, in human AM compared with PBM [9]. The upregulation of 5-LO metabolism appears to occur in the alveolar space, since pulmonary interstitial macrophages display an eicosanoid profile similar to that of nonpulmonary macrophages [10]. Many investigators have studied the mechanisms by which PBM differentiate into AM and factors involved in changes in AA metabolic patterns. Nonpulmonary macrophages cultured over time with or without increased oxygen tension failed to increase 5-LO metabolism [10]. Culturing PBM with surfactant alone tended to be toxic, and suppressed AA release (unpublished data). Incubation of PBM with vitamin D increased LT synthetic capacity in parallel with an increase in FLAP expression [17]. Other investigators have demonstrated increased 5-LO activity in PBM incubated with the cytokine granulocyte macrophage colony-stimulating factor (GMCSF) [18]; however, in the authors' experience GM-CSF did not affect PBM eicosanoid generation or alter 5-LO or FLAP expression [12]. Interferon- $\gamma$ increased the LT synthetic capacity of AM, without any effect on AA release or new protein synthesis [19]. While it would be desirable to be able to study factors individually, it is likely that the complexity of the system is such that it cannot be mimicked by any single mediator or condition. The approach in this study starts broad and provides the foundation for future studies seeking to home in on specific factors in ALF which upregulate mononuclear phagocyte LT synthetic capacity.

The hypothesis that ALF, which bathes the cells located in the alveolus, would enhance 5-LO metabolism in PBM recruited into the lung and maintain the heightened 5-LO metabolic capacity observed in AM was tested. Firstly, the model of human PBM entering the lung and differentiating into AM was examined. Human PBM were investigated because of their far greater availability than rat PBM, which are difficult to obtain in high enough numbers for metabolic and protein studies. Furthermore, human PBM a)
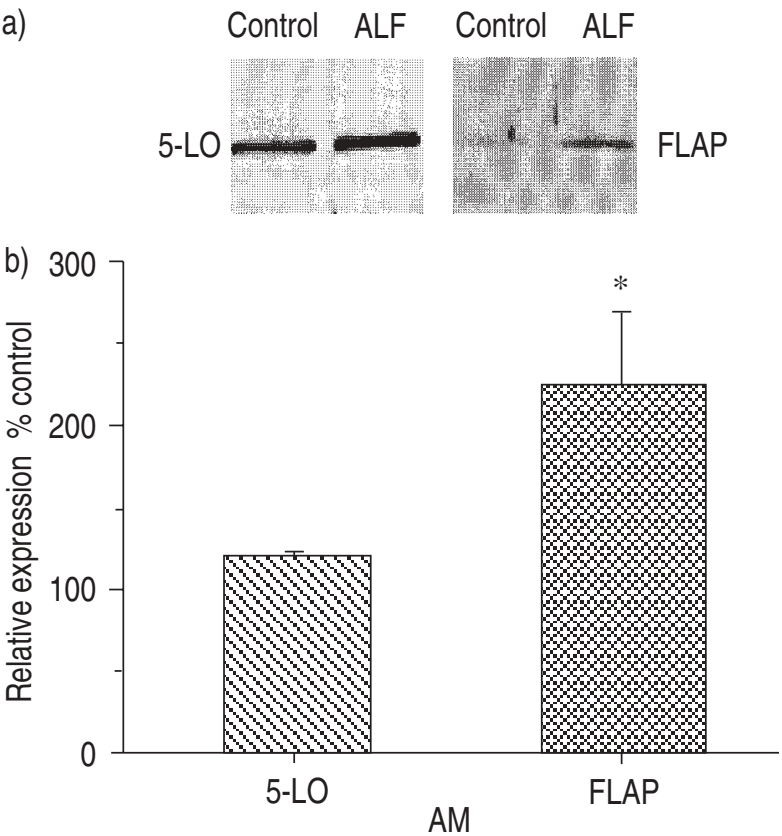

Fig. 5. - Effect of alveolar lining fluid (ALF) on 5-LO activating factor (FLAP) expression in alveolar macrophages (AM). AM were cultured with or without ALF in the absence of serum overnight. Western blot analysis was performed, as described in figure 2. a) Representative autoradiograph of a Western blot demonstrating the amount of 5-LO (left) and FLAP (right) in AM incubated with or without ALF. b) Comparison of the relative expression of 5-LO and FLAP in AM cultured with and without ALF, as assessed by densitometry and expressed as a percentage of values derived from control cells run in parallel. Data represent the mean \pm SEM from three experiments. *: $\mathrm{p}<0.05$ compared with control cells.

are similar to rat PM in that their 5-LO activity and 5-LO and FLAP expression decline in culture over time [20]. ALF obtained from rats was utilized because of a number of problems associated with human ALF. Samples of ALF obtained from humans are very dilute because of the large volumes of lavage used. In addition, human ALF is not as suitable for culturing cells, since preliminary experiments with human ALF resulted in clumping of macrophages in culture. This observation may be explained by the presence of an endotoxin.

Although AM have higher levels of 5-LO than PBM [9, $21]$ the greater 5-LO metabolic capacity relates more to their very increased level of FLAP expression. If this increase in FLAP expression is a consequence of residence in the alveolar space, the effects of ALF would be expected similarly to increase FLAP more than 5-LO expression. This was indeed the finding. There was, correspondingly, a greater decrease in FLAP expression in culture over several days in AM than in PM. This observation fits with a gene promoter analysis indicating that 5-LO is a constitutively expressed protein, while FLAP is subject to induction [22]. One factor which is known to increase LT synthesis and FLAP expression in parallel is 1,25-dihydroxyvitamin $\mathrm{D}_{3}[17,23]$. Furthermore, 1,25 -dihydroxyvitamin $\mathrm{D}_{3}$ is present in ALF in greater concentrations than in plasma [9] and 5-LO metabolism and FLAP expression were decreased in AM from vitamin D-deficient rats.

The above observations suggest that culturing AM in ALF, containing mediators that better approximate the true culture environment for macrophages resident in the alveolus, results in persistent expression of FLAP. However, 
there was a relatively modest effect of ALF on 5-LO product formation compared with the effect on 5-LO and particularly FLAP expression. There was a trend towards a decrease in AA release, which may reduce LT synthesis relative to 5-LO and FLAP expression. In addition, as well as a need for soluble mediators there may be a requirement for specific cell-to-cell interactions to maximize 5-LO product formation. Studies are ongoing to determine the optimal concentration of ALF which upregulates 5-LO metabolism, and protein expression. Furthermore, addition-al studies will be performed to identify the specific factor(s) secreted into ALF that may be responsible for this increase in 5-LO metabolism.

In conclusion, it was demonstrated that factors present in alveolar lining fluid upregulate 5-lipoxygenase metabolism and 5-lipoxygenase and 5-lipoxygenase activating protein expression in peripheral blood monocytes and alveolar macrophages. In view of the importance of leukotrienes in the phagocytosis and killing of micro-organisms, upregulation of leukotriene synthesis may be important for optimal antimicrobial function of macrophages defending the alveolar space [24, 25]. Defects in mononuclear phagocyte 5-lipoxygenase metabolism occur in a variety of conditions associated with increased susceptibility to pulmonary infection, including malnutrition [26], vitamin deficiency [14] and infection with human immunodeficiency virus [27]. One clinical implication of these investigations is the development of possible strategies to increase 5-lipoxygenase metabolism and therefore boost host defence capabilities.

\section{References}

1. Rouzer C, Ford-Hutchinson A, Morton H, Gillard J. MK886, a potent and specific leukotriene biosynthesis inhibitor blocks and reverses the membrane association of 5-lipoxygenase in ionophore-challenged leukocytes. $J$ Biol Chem 1990; 265: 1436-1442.

2. Israel E, Dermarkarian R, Rosenberg M, et al. The effects of a 5-lipoxygenase inhibitor on asthma induced by cold, dry air. N Engl J Med 1990; 323: 1740-1744.

3. Garcia J, Griffith D, Cohen A, Callahan K. Alveolar macrophages from patients with asbestos exposure release increased levels of leukotriene $\mathrm{B}_{4}$. Am Rev Respir Dis 1989; 139: 1494-1501.

4. Wilborn J, Bailie M, Coffey M, Burdick M, Strieter R, Peters-Golden M. Constitutive activation of 5-lipoxygenase in the lungs of patients with idiopathic pulmonary fibrosis. J Clin Invest 1996; 97: 1827-1836.

5. Fels A, Pawlowski N, Cramer E, King T, Cohn Z, Scott $\mathrm{W}$. Human alveolar macrophages produce leukotriene $\mathrm{B}_{4}$. Proc Natl Acad Sci USA 1982; 79: 7866-7870.

6. Fels A, Cohn Z. The alveolar macrophage. J Appl Physiol 1986; 60: 353-369.

7. Bigby T, Holtzman M. Enhanced 5-lipoxygenase activity in lung macrophages compared to monocytes from normal subjects. J Immunol 1987; 138: 1546-1550.

8. Balter M, Toews G, Peters-Golden M. Different patterns of arachidonate metabolism in autologous human blood monocytes and alveolar macrophages. J Immunol 1989; 142: 602-608.

9. Coffey M, Wilcoxen S, Peters-Golden M. Increases in 5-lipoxygenase activating protein expression account for enhanced capacity for 5-lipoxygenase metabolism which accompanies differentiation of peripheral blood monocytes into alveolar macrophages. Am J Respir Cell Mol Biol 1994; 11: 153-158.
10. Peters-Golden M, McNish RW, Hyzy R, Shelly C, Toews GB. Alterations in the pattern of arachidonate metabolism accompany rat macrophage differentiation in the lung. J Immunol 1990; 144: 263-270.

11. Sporn P, Murphy T, Peters-Golden M. Complex effects of in vitro hyperoxia on alveolar macrophage arachidonic acid metabolism. Am J Respir Cell Biol 1990; 2: 81-90.

12. Brock T, McNish R, Coffey M, Clark Ojo T, Phare S, Peters-Golden M. Effect of granulocyte-macrophage colony- stimulating factor on eicosanoid production by mononuclear phagocytes. J Immunol 1996; 156: 2522-2527.

13. Coffey M, Peters-Golden M, Fantone J, Sporn P. Membrane association of active 5-lipoxygenase in resting cells: evidence for novel regulation of the enzyme in the rat alveolar macrophage. J Biol Chem 1992; 267: 570-576.

14. Coffey M, Wilcoxen S, Simpson R, Phare S, Gyetko M, Peters-Golden M. Reduced 5-lipoxygenase metabolism in macrophages from 1,25-dihydroxyvitamin $\mathrm{D}_{3}$-deficient rats. Prostaglandins 1994; 48: 313-329.

15. Laemmli U. Cleavage of structural proteins during the assembly of the head of bacteriophage $\mathrm{T}_{4}$. Nature 1970; 227: 680-685.

16. Wilborn J, DeWitt DL, Peters-Golden M. Expression and role of cyclooxygenase isoforms in alveolar and peritoneal macrophages. Am J Physiol 1995; 268: L294-L301.

17. Coffey M, Gyetko M, Peters-Golden M. 1,25-Dihydroxyvitamin $\mathrm{D}_{3}$ upregulates 5-lipoxygenase metabolism and 5lipoxygenase activating protein in peripheral blood monocytes as they differentiate into mature macrophages. $J$ Lipid Med 1993; 6: 43-51.

18. Ring W, Riddick C, Baker J, Munafo D, Bigby T. Lymphocytes stimulate expression of 5-lipoxygenase and its activating protein in monocytes in vitro via granulocyte macrophage colony-stimulating factor and interleukin 3. J Clin Invest 1996; 97:1293-1301.

19. Meslier N, Aldrich A, Bigby T. Effect of interferongamma on the 5-lipoxygenase pathway of rat lung macrophages. Am J Respir Cell Mol Biol 1992; 6: 93-99.

20. Ring W, Riddick C, Baker J, Munafo D, Bigby T. Human monocytes lose 5-lipoxygenase and FLAP as they mature into monocyte- derived macrophages in vitro. Am J Physiol 1996; 271: C372-377.

21. Pueringer R, Bahns C, Hunninghake G. Alveolar macrophages have greater amounts of the enzyme 5-lipoxygenase than do monocytes. J Appl Physiol 1992; 73: 781-786.

22. Kennedy B, Diehl R, Boie Y, Adam M, Dixon R. Gene characterization and promoter analysis of the human 5lipoxygenase-activating protein (FLAP). $\mathrm{J}$ Biol Chem 1991; 266: 8511-8516.

23. Bennett C, Chiang M, Monia B, Crooke S. Regulation of 5-lipoxygenase and 5-lipoxygenase-activating protein expression in HL-60 cells. Biochem J 1993; 289: 33-39.

24. Demitsu T, Katayama H, Saito-Taki T, Yaoita H, Nakano M. Phagocytosis and bactericidal action of mouse peritoneal macrophages treated with leukotriene $\mathrm{B}_{4}$. Int J Immunopharmacol 1989; 11: 801-808.

25. Bailie M, Standiford J, Laichalk L, Coffey M, Strieter R, Peters-Golden M. Leukotriene-deficient mice manifest enhanced lethality from klebsiella pneumonia in association with decreased alveolar macrophage phagocytic and bactericidal activities. J Immunol 1996; 157: 5221-5224.

26. Skerrett S, Henderson W, Martin T. Alveolar macrophage function in rats with severe protein calorie malnutrition: arachidonic acid metabolism, cytokine release, and antimicrobial activity. J Immunol 1990; 144: 1052-1061.

27. Coffey M, Phare S, Kazanjian P, Peters-Golden M. Reduced 5-lipoxygenase metabolism in alveolar macrophages from subjects infected with the human immunodeficiency virus. J Immunol 1996; 157: 393-399. 